\%. Phon. Sprachwiss. Kommun.forseh. (ZPSK), Berlin 42 (1989) 6, 715-721

HANS-Petwir VIETZE (Ber]in)

\title{
Zur Struktur des Wortes in altaischen Sprachen
}

\section{Summary}

In continuation of two previous articles in which the word and its scmnntic structure in Altaic langunges were discussed the word is regarded as a set of monemes. 'This set is dissected in different ways, especially under the aspect of the positional distribution of the monemes. 'The result is a model in the form of a tree ending in a string. This string reflects the potential morphologien! structure of any word of an Altaic langunge.

\section{Corpus}

Unter dem Begriff „altaische Sprachen“" werden die Turksprachen sowie die mongolischen und mandschurisch-tungusischen Sprachen zusammengefaßt, woriuber unter den Fachwissenschaftlern im wesentlichen Eimmütigkeit besteht. Umstritten ist die Zugehörigkeit des Koreanischen und Japanischen zu dieser Sprachfamilie. Das P.'roblem der genetischen Verwandtschaft der altaischen Sprachen, die sogenamnte altaische Hypothese, bleibt unbewiesen und vielleicht mobeweisbar, wenn man bedenkt, daß die ältesten alttïrkischen Inschriften, die aus dem 8. Jh. stammen, fast 3000 Jahre jünger sind als die ältesten bekannten indoemropäischen Quellen.

'I'ypologisch vereinen die altaischen Sprachen in erster Linie die in der Morphologie stark dominierende Agghutination sowie in der Syntax das ,altaische Grundprinzip“ (das Bostimmende steht vor dem zu Bestimmenden). Es gibt nur wenig eindeutige intersprachliche Lautgesetze. Die typologischen Gemeinsamkeiten sind jedoch groß genug, um likenntnisse zu einer Sprache in viclen Fällen auf eine andere transponieren zu können, wie auch im folgenden gezeigt werden soll, so da B die mit diesen Sprachen befaßte orientalistische Teildisziplin, die Altaistik, zumindest pragmatisch gerechtfertigt ist.

\section{Honeme und Morpheme}

Wir hatten in friiheren Aufsätzen versucht, das mongolische Wort über cinen Algorithmus zu definieren 1 und ein Regelsystem zu erarbeiten, das jedes geschriebene Wort einer altaischen Sprache in Moneme zerlegt,2 das tiirkische Wort dostlarma 'meinen Fremnden' also z. B. in die Monemfolge dost|lar $/ \mathrm{m} \mid \mathrm{a}$. Heuristisch seien die Moneme als kleinste bedeutungstragende Einheiten umrissen, definiert sei eine endliche Menge $A$ aller Moneme $a_{i}(1 \leq i \leq n)$ über das $0 . \mathrm{g}$. Regelsystem oder über eine Iiste. 
Aus $A$ wird eine echte Teilmenge $B$ ausgesondert: $A \supset B . B$ ist die Menge aller Moneme $a_{i}$, für die ein $a_{j}$ existiert, wobei gilt: $\iota_{i} \neq a_{j}$ und $a_{i} \approx a_{j}$. Die Äquivalenzrelation $\approx$ definiert in $B$ eine Verlegung in parweise disjunkte Klassen $C_{i}(1 \leq i \leq k)$, deren Vereinigung wieder gleich der Menge $B$ ist. Eine Klasse $C_{i}$ ist eine aus mindestens zwei Elementen bestchende Menge semantisch gleichwertiger Moneme in komplementärel Distribution.

Beispiel für eine Klasso $C_{1}$ (mong.): malyn 'des Viehs', modny 'des Bammes', gerijn 'dor' Jurte', cajn 'des T'Tes', chödö̈nij oder chödöögijn 'der ländlichen Gegend'. Die sechs unterstrichenen Moneme sind semantisch gleichwertig (sie bilden den (Genitiv). Ihre Auswahl wird vom Vokalbestand und vom Auslaut des jeweiligen Wortstammes bestimmt. Wine Klasse $C_{1}$ kann auch nur zwei Elemente enthalten, z. B. (mong.) ci 'du' und cam 'du' (in čam šig 'wie du').

Jede Klasse $C_{1}$ heißt Morphem, jedes Element einer Klasse $C_{1}$ heilt Allomorph. Morpheme sind Mengen von mindestens zwei Monemen. Allomorphe sind Moneme. Die Teilmenge $B \subset A$ ist die Menge aller Morpheme der untersuchten Sprache.

Ein Morphem wird im folgenden als fiir $C_{i}$ repräsentatives Allomorph zwischen Strichen |... notiert, da die iihliche Notation (geschweifte Klammern) zu Verwechslungen mit der mengentheoretischen Symbolik führen könnte. Bei Schwierigkeiten in Ausnahmefällen, wie z. B. tiirk. eski 'alt' - epeski 'ganz alt', rrak 'fern' spirak 'ganz fern', uzun 'lang' - upuzun 'ganz lang' (ep, zp und up sind per definitionem Allomorphe), kamn das Morphem iiber eine Konvention als $\left|x_{1} p\right|$ notiert werden ( $x_{1}$ : erster Buchstabe).

\section{Lexeme und Suffixe}

Neben der Aussonderung der Teilmenge $B$ kann die Menge $A$ (die Menge aller Moneme) in zwei Klassen $D$ und $E$ mit den Bedingungen $D, E \subset A, D \cup E=A$ und $D \cap E=0$ zerlegt werden. Die Elemente von $D$ heißen Lexeme. Ein Lexem ist ein isolierbares, autosemantisches Monem, z. B. (1) tiirk. ev 'Haus', (2) mandsch. monggo|so 'Mongolen', (3) mong. av|c|ir 'bringe herbei!'. J) eines Wortes mit zwei getrennten Iexemen (av 'nimm!', ir 'komme!', ¿: Konverb. kennzeichnung).

Die Klasse $E$ kann leicht als die I Differenz $A \backslash D$ definiert werden, d. h. jedes Monem, das nicht Element der Klasse $I$ ) (kein Lexem) ist, ist ein Element der Klasse $E: a \in E$ genau dann, wenn $u \notin D$ ( $a$ : Monem). Die Elemente der Klasse $E$ heißen Suffixe. Beispiele für Suffixe sind die nicht unterstrichenen Moneme in den o. a. Beispielen (2) und (3).

Lemma: Die Yerlegung der Menge $A$ in die Klassen $D$ und $E$ ist keine Verfeinerung der Zerlegung der Menge $B$ in die Klassen $C_{1}$, denn wem ein beliebiges Element eines Morphems ein Suffix ist, dann sind alıch alle übrigen Elemente dieses Morphems Suffixe.

$C_{i} \subset(D \cap B)$ (eine Menge, die sich im Durchschnitt $(D \cap B)$ befindet) heiBt LexemMorphem, $C_{i} \subset(E \cap B)$ heißt Suffix-Morphem. Beispiel für ein Lexem-Morphom: mong. $|\mathbf{r u u}|=$ ruu, rüü, luu, lüü 'in Richtung auf . . . Beispiel für ein Suffix-Morphem: mandsch. $|-n a|=-n a,-n e,-n o$ (Illativ). 


\section{Wortbildende und formbildende Suffixe}

Die Menge $E$ aller Suffixe kann in zwei Klassen $F$ und $G$ zerlegt werden. Die Wlemente der Klasse $F$ heißen wortbildende Suffixe, die Elemente der Klasse $G$ heißen formbildende Suffixe. Ein Suffix gehört zur Klasse $F$, d. h. es ist wortbildend, wem es potentiell nur genau die Bedentung desjenigen Wortstammes, an den es angefiigt ist, zu einer nenen Wortbedentung transformiert, z. B. (mong.): temee|c|in aduu $\ddot{c} \mid$ in chojor 'der Kamelhirt und der P'ferdehirt'

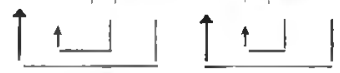

1)ie Suffixe $-\check{c}$ und - in miissen sowohl an das Wort temee 'Kamel' als anch an das Wort adum 'Pferde(-herde)' angefügt werden, um neue Wörter mit der Bedeutung 'Kanelhirt' bzw. 'Pferdehirt' zu erhaiten. Bei verbalen Suffixen wird dieser Test mit Hilfe von konverhen durchgefiilurt, z. B.:

Bi tüï|ijg san $u$ ulaad jav|uul| san. 'Jch habe ihn (daran) erinnert und wegge$\uparrow$ † l schiclit.'

Das Kausativ-Suffix -unl muk sowohl an das Icexem sana- 'sich erimem, als auch an das Lexem jav- 'gehen' angefügt werden, um die Bedentungen 'erimem' bzw. 'wegschicken' zu erhalten.

Die Menge $F$ aller wortbildenden Suffixe kann nach morphologischen, syntaktischen semantischen, phonetischen 1 . a. Kriterien in eine grobe \%ahl von Klassen, z. B. in Klassen deverbaler, verbbildender, hintervokalischer I. a. Suffixe zerlegt werden, was in jeder Grammatik getan wird. Im folgenden wird die Menge $\boldsymbol{F}$ nach der Positjon der worthildenden Suffixe in Klassen FS zerlegt. Diese Klassen versehen wir mit dem Positionsindex $\left({ }_{1}\right)$.

$F S_{(1)}$ : Dje worthildenden Suffixe dieser Klasso nehmen in der Suffixkette eines beliehigen Wortes potentiell den ersten Platz ein, a. h. sie stehen nach cinem hexem, z. B. mandsch. -la in aisilla- 'helfen'.

$F S_{(i)}$ : Die wortbildenden Suffixe dieser Klassen $(1 \leq i \leq n)$ können an lexeme oder all wortbildende Suffixe der Klassen $F S_{(1)}, F S_{(2)}, \ldots, F S_{(i-1)}$ angefügt verden, \%. 3. mandsch. -ndu in aisilalndu- 'einander' helfen'.

FS $S_{(u)}$ : Die wortbildenden Sutfjxe dieser Klasse stehen in ler Kette der wortbildenden Suffixe cines beliebigen Wortes an letzter Position. Das gilt z. B. für die Nomina actoris bildenclen mongolischen Suffixe |-age oder fiir die türkische Negation |-ma|.

Swify war unseres Wissens der erste, der die tiurkischen Suffixe zunächst nach traditionellen Gesichtspunkten, dam aber auch nach ihrem o. a. positionellen Verhalten klassifizierte." Hier sei sein Beispiel kosișitur ulma- "nicht miteinander herumronnen lassen' angefialu't

\begin{tabular}{lllll} 
Stamm & 1 & 2 & 3 & \pm \\
& $\begin{array}{l}\text { reflexiv } \\
\text { reziprok }\end{array}$ & liansativ & jussiv & Negation \\
\hline $\begin{array}{l}\text { koş- } \\
\text { rennen' }\end{array}$ & -"ş & -tur & - -ul & -ma
\end{tabular}

Die Klasse $G$, deren Elemente formbildende Suffixe heißen, wird wie folgt abgegrenzt: Ein Suffix gehört zur Klasse $G$, wenn sein semantisches Wirkungsbereich ïber das Wort, dessen Bestandteil es ist, hinausgehen kann und ein oder mehreve gleichgeord- 
nete vorhergehende Wörter mit umfaßt, z. B. (mong.): ech ür| ïn žargal 'das Gliick der Mutter und des Kindes'

Das Suffix -ijn (Genitiv) ist nur an das Lexem ïr 'Kind (lit.)' angefügt, erstreckt sich aber in seiner grammatischen Bedeutung auch auf das vorangegangene gleichgeordnete Wort ech 'Mutter'. Bei verbalen Suffixen wird der 'Test wieder mit Hilfe von Konverben durchgefïhrt, z. B.:

Dorz chödöö jav|̌̆ amar|na. 'Dorž wird auf das Land fahren und sich erholen.'

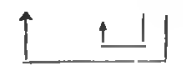

Das Suffix -na (hier: Futur) erstreckt sich in seiner grammatischen Bedeutung sowohl auf das Lexem amar- 'sich erholen' als auch auf das Lexem jav - (hier): fahren'.

Die Menge $G$ der formbildenden Suffixe kann unter dem Gesichtspunkt ihres positionellen Verhaltens wieder in Klassen $G S_{(i)}(1 \leq i \leq n)$ zerlegt werden.

$G S_{(1)}:$ Die formbildenden Suffixe dieser Klasse stehen immer unmittelbar nach I.exemen oder worthildenden Suffixen, z. B. mong. Pl. -d in nöchö(r)|d 'Genossen'.

$G S_{(2)}$ : Nach Lexemen, wortbildenden Suffixen und formbildenden Suffixen der

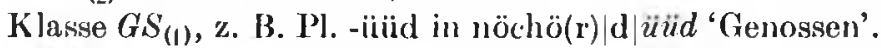

$G S_{(i)}$ : Wie $G S_{(2)}$, aber auch nach formbildenden Suffixen der Klassen $G S_{(2)}, \ldots, G S_{(i-1)}$, z. B. Genitiv -ijn in nöchö(r)|d|üïd $\mid \ddot{j} n$ 'der Genossen', Isokativ -d in nöchö(r)|dïüd|ijn|d 'bei den Genossen'.

$G_{(n)}$ : Am Ende eines Wortes, z. B. Possessiv -ee in nöchö(r)|d|iiüd|ijn|d|ee 'bei seinen Genossen'.

Jie Mengen $F$ und $G$ können zumindest für die mongolische Sprache nicht als disjunkt betrachtet werden. Die Elemente (Suffixe) im Durchschnitt $F \cap G$, deren Zahl sehr gering ist, kömmen sowohl wort- als nuch formbildend sein, z. B.:

nöchör saj(n)|d 'Genosse Minister' (nöchör 'Genosse', sajn 'gut', sajd 'Minister', -d $\uparrow$ wortbildend, hier: Pluralis majestatis).

gachaj nocho(j)|d 'Schweine und Hunde' (gachaj 'Schwein', nochoj 'Hund', -d Plural,

$\uparrow \quad$ f formbildend).

Im Tïrkischen ist diese Ėrscheinung unseres Wissens nicht bekannt, wohl aber im Mandschurischen: bei| se 'Beise' (Titel). -se ist analog zu mong. -d an saij(n) ein hier wortbildendes Suffix.

Im weiteren betrachten wir wieder die Relationen zwischen den nicht disjunkten Klassen $F$ und $G$ und der Teilmenge $B\left(B \subset A, B=C_{1}, C_{2,}, \ldots, C_{k}\right)$. Parallel zur Definition der Lexem-Morpheme und der Suffix-Morpheme bezeichnen wir alle Mengen $C_{1}$ im Durchschnitt $F \cap B$ als wortbildende Suffix-Morpheme, alle $C_{f}$ im Durchschnitt $G \cap B$ als formbildende Suffix-Morpheme und alle Mengen $C_{h}$ im Durchschnitt $(F \cap G) \cap B$ als Suffix-Morpheme, die gleichzeitig wort- und formbildend sind. Beispiel für ein wortbildende Suffix-Morphem: mandsch. $|-l a|=-l a,-l e,-l o,-r a,-r e,-r o$ (denominales V'erbbildungssuffix). Beispiel für ein formbildendes Suffix-Morphem: mandsch. $\mid$-sa $\mid=-s a$, -se (Pluralsuffix). Beispiel für ein sowohl wort- als auch formbildendes Suffix-Morphem: mong. $\mid$-nar $\mid=-$ nar, -ner (formbildend als Pluralsuffix, wortbildend an ech 'Mutter' : echner 'Gattin').

Alle Elemente im Durchschnitt $F \cap B$ heißen wortbildende Suffix-Allomorphe, alle Elemente im Durchschnitt $G \cap B$ formbildende Suffix-Allomorphe und alle Elemente in Durchschnitt $(F \cap G) \cap B$ Suffix-Allomorphe, die gleichzeitig wort- und 
formbildend sind. Alle diese Elemente sind Moneme. Als Allomorphe von SuffixMorphemen werden sie nur dann besonders ausgewiesen, wemn ihre Relation zur Teilmenge $B$ zu diskutieren ist.

\section{Darstellung der Wortstruktur}

Jedes Wort einer altaischen Sprache kann in eine Lexemkonfiguration $K L$ und in eine Suffixkonfiguration $K S$ zerlegt werden, wobei nur $K S$ leer sein darf:

$\mathbb{W}==\Rightarrow K L, K S$

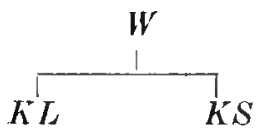

Betrachten wir zunächst die Lexemkonfiguration $K L$. Sie beginnt nach einem $Z w i s c h e n r a u m$ und endet mit dem im Wort am weitesten rechts stehenden Lexem $L$. Folgende Strukturen von $K L$ sind möglich:

$$
K L==\Rightarrow L \quad K L==\Rightarrow L_{1}, L_{2} \quad K L==\Rightarrow L_{1}, S, L_{2} \quad \text { (S: Suffix) }
$$

Somit erhalten wir für die potentielle Lexemkonfiguration $K L$ und ihre Relation zum Wort $W$ folgenden Baum:

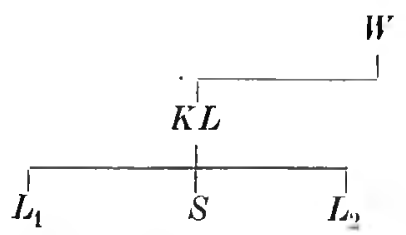

Lemma: Da per definitionem $K L$ mit dem im Wort am weitesten rechts stehenden Lexem endet, kamn ein Suffix in $K L$ nur damn auftreten, wenn ein weiteres Lexem noch nach diesem Suffix folgt.

Die Suffixkonfiguration $K S$ kann in die Konfiguration der wortbildenden Suffixo $K F S$ und in die Konfiguration der formbildenden Suffixe KGS zerlegt werden:

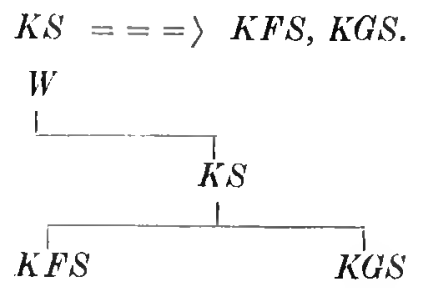

$K F S$ beginnt unmittelhar nach der Lexemkonfiguration $K L$ und endet mit dem im Wort am weitesten rechts stehenden wortbildenden Suffix.

KF'S kann $u$ wortbildende Suffixe enthalten, dio nach den Indices der Positionsklassen, zu denen sie gehören, geordnet werden:

$K F S==\Rightarrow F S_{(1)}, F S_{(!)}, \ldots, F S_{(n)}$ (Bsp. s. Abschnitt 4). 


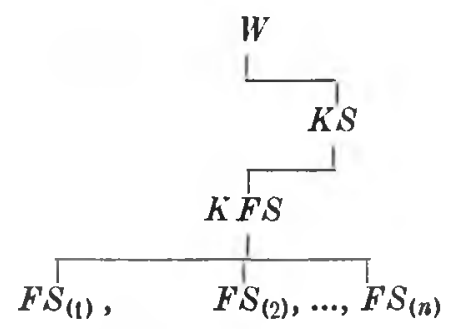

Die Konfiguration der formbildenden Suffixe $K G S$ beginnt unmittelbar nach $K F S$ oder nach der Lexemkonfiguration $K L$, wenn im konkreten Fall $K F S$ leer ist. $K G S$ enthält $n$ formbildende Suffixe, die wieder ihrem positionellen Verhalten gemäßgeordnet sind :

$K G S===G S_{(1)}, G S_{(2)}, \ldots, G S_{(n)}$ (Bsp. s. Abschnitt 4).

W

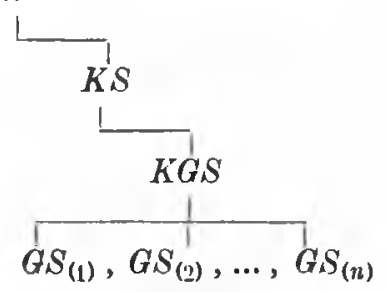

Diejenigen Suffixe, die sowohl wort- als auch formbildend sein können, verhalten sich neutral, d. h. sie können sowohl den Platz von wortbildenden Suffixen in KFS als auch den Platz von formbildenden Suffixen in KGS eimnehmen.

Das formale Gesamtmodell für das morphologische Grundinventar des Wortes und sein positionelles Verhalten stellt sich wie folgt dar:

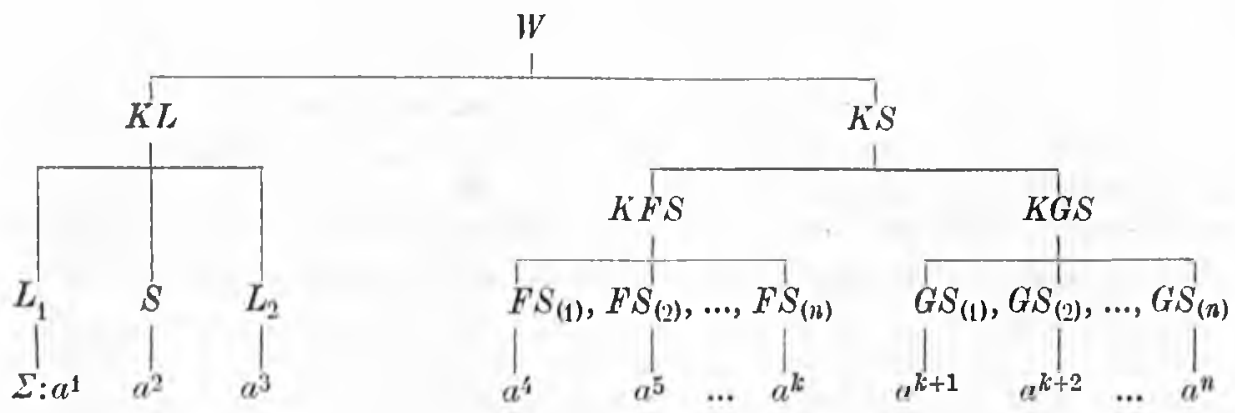

Jedes Wort einer altaischen Sprache kann eindeutig in die Endkette $\Sigma$ abgebilclet werden, wobei im Spezialfall, d. h. bei einer altaischen Sprache, die uns nicht bekannt ist, eine Mlodifizierung des Modells erfolgen kann. Eine eincindentige Abbildung kommt in praxi nicht vor. Die Urbilder der Abbildung sind die Moneme $a_{1}, a_{2}, \ldots, a_{16}$ eines beliebigen Wortes einer altaischen Sprache, jhre Bilder sind die Elemente $a^{1}$, $a^{2}, \ldots, a^{n}$ der Fndkette $\Sigma$.

Für die Abbildung gelten die folgenden Regeln (1) und (2):
(1) $a_{1} \rightarrow a^{1}$
(2) $u_{i}(1 \leqq i \leq n) \rightarrow a^{j}(j \geq i, 1<j \leq n)$

d. $h$. jedes erste Monem $a_{1}\left(a_{1} \in W\right)$, das per definitionem ein Lexem sein muß, ist 
$a^{1}\left(a^{1} \in \Sigma\right)$ zuzuordnen. Jedes folgende Monem $a_{i}(i>1)$ wird einem Element $a^{j}$ aus $\Sigma$ zugeordnet, wobei $a^{j}$ denselben oder einen höheren Jndex hat als $a_{i}, z$. B.:

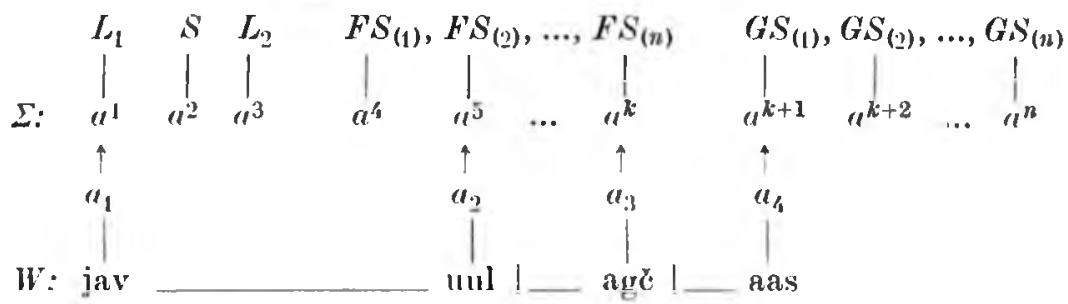

'vom Absender' (mong.), __ : Uberbriickung der grafisch bedingten Zwischenräune.

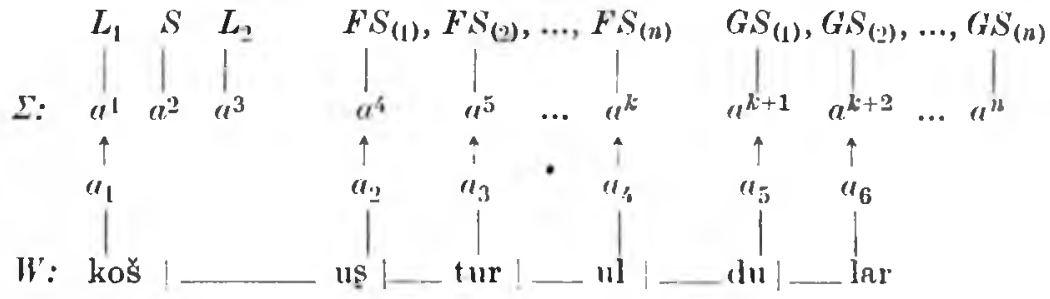

Sie haben herumrennen lassen' (türk.)

Demgegenüber ist eine Abbildung wie z. B.

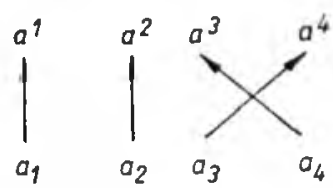

nicht zugelassen.

Danit hahen wir die Grmbrige eines Jodells erarbeitet, dns das morphologische Grundinventar des Wortes formal und positionell im wesentlichen erfa Bt und zuminlest für die wichtigsten altaischen Sprachen guiltig ist. Fïr andero altaische Sprachen, die hier nicht berücksichtigt werden komnten, kamn es entsprechend modifiziert werden. Die anfwendig erscheinende Struliturierung des Wortes ist durch die ansgeprägto Agglutination in den altaischen Surachen gerechtfertigt.

\section{Jiteratur}

VIs're, H.-P., Eine Defintion des mongolischen Wortes, in: ZJPK, Bd. 22 (1969), Heft 1, S. $77-82$.

-, Dens Iroblem der Semantikstrukturierung bei eincr automatischen monematischen Analyse, in: Z $Z$ PSK, Bd. 23 (1971), Heft 6, S. 590-615.

SwIT, L. B., A Reference Grammar of Modern T'urkish, Indiana L'niversity I'ublications, Uralic and Altuic Scries, Vol. 19, I)en Hang 1963, S. 114.

Prof. Dr. Hans-Peten Viftze, Humboldt-Universitït zu lierlin, Soktion Asienwissenschaften 